\title{
Invasive Lower Lip Squamous Cell Carcinoma Mimicking Traumatic Fibroma: Case Report
}

\author{
Venkatesh Hange ${ }^{1}$ \\ ${ }^{1}$ Junior Resident, Department of Oral and Maxillofacial Surgery, K.D. Dental College \& Hospital, \\ Mathura, Uttar Pradesh, India.
}

\author{
Article Info \\ Article history: \\ Received: 13 September 2020 \\ Revised: 28 October 2020 \\ Accepted:05November 2020

\section{Keywords:} \\ Lower Lip Cancer, \\ Squamous Cell Carcinoma, \\ Differential Diagnosis, \\ Elective Neck Dissection, \\ Mimicking Lesion. \\ Paper Type : \\ Case Report \\ Corresponding Author: \\ Venkatesh Hange \\ Email: \\ venkateshhange@yahoo.com
}

\begin{abstract}
Carcinoma of lip develops due to formation of malignant cells in the lips and it is the most common site for squamous cell carcinoma (SCC) in the head and neck region. Oral cavity is among top five leading sites of cancer, regardless of gender distribution across India. Squamous cell carcinoma of lip occurs at myriad locations along the upper or lower lip or commissure of lip, but majority ( $80 \%$ to $95 \%$ ) of cases are seen on the lower lip region. Majority of oral cavity carcinoma cases (> 90\%) are seen in patients above the age of 45, having a male predilection in distribution. Lesions that includes up to one third of the lower lip are typically treated with V-type or $W$-type excisions. Lesions affecting between one to two thirds of the lower lip are typically treated by regional flaps of Abbe or Estlander. Larger lesions involving more than two thirds of the lower lip are treated with Bernard flap for middle lesions and nasolabial transpositional flap for lateral lesions. Alternatively, definitive radiotherapy can be used for local control of early-stage epithelial malignancies of the lips such as basal cell and squamous cell carcinomas, but usually reserved for selected situations, also side effects of irradiation limits its use. Local resection along with elective SOHND is mandatory for long term survival rate and to avoid substantial worse prognosis. This case report describes an unusual presentation of carcinoma of lip mimicking benign soft tissue swelling.
\end{abstract}

\section{Introduction}

Carcinoma of lip is a condition which develops due to the formation of malignant cells. The most common in head and neck region is the squamous cell carcinoma (SCC). Oral cavity is among the top five leading sites of cancer regardless of gender distribution across India. The majority of oral cavity carcinoma (>90\%) is seen in patients above the age of 45 , having a male predilection in distribution. The occurrence of SCC increases steadily with age until 65 years of age, achieving its pinnacle between the ages of 70 to 80 years (Shafer et al., 2006). The lower lip is affected far more frequently ( $80 \%$ to $95 \%)$ than the upper lip (2\% to $12 \%$ ) or commissure (1\% to $15 \%$ ). The majority of SCC of the lower lip stems from the vermilion border (Duzgun et al., 2013).

Nearly $90 \%$ tumours found on lip consist of squamous cell carcinomas (SCC) and comparatively much lesser cases include basal-cell tumors (BCC). However, some adenocarcinomas are derived from the minor salivary glands and, even more rarely, melanomas, sarcomas and lymphomas may occur. Basal-cell tumors are more prevalent in the upper lip than the lower lip and rarely undergo lymph node metastasis (Zitsch et al., 1999). 
Prognosis and survival factor of carcinoma of lip to a major extent depends upon cervical Lymph Node Metastasis (LNM). Lip SCC develops LNM in the range of 6 to 37\% even late lymph node metastases can be seen in $8 \%$ patients of lip SCC if Elective neck dissection (END) is excluded from the treatment protocol. It seems that $25 \%-50 \%$ patients survive beyond five years postoperative. Therefore, to avoid unfavourable prognosis, early detection of occult LNM lip cancers is crucial (Salgarelli et al., 2009).

This case report describes an unusual presentation of carcinoma of lip mimicking a benign soft tissue swelling, considering the life threatening potential of squamous cell carcinoma, while making a differential diagnosis of inflammatory swellings of lip, squamous cell carcinomas should not be ruled out.

\section{Case Report}

A 55 year old male patient reported with the chief complaint of proliferating growth in the lower lip (Fig.1). The patient became aware of this swelling 2-3 months before seeking medical help. Any forms of discomfort due to proliferation were absent. The patient had a history of tobacco chewing for the last 18 years and was a chronic alcoholic. The patient had a tendency to hold the tobacco quid in the buccal vestibule. The patient also gave a history of extraction of proclined maxillary anterior teeth 3 weeks back as the maxillary anterior teeth were thought to be the cause of traumatic swelling on the lower lip by a private dental consultant. Extraoral examination revealed sub-mandibular lymph nodes palpable on right side of the face.

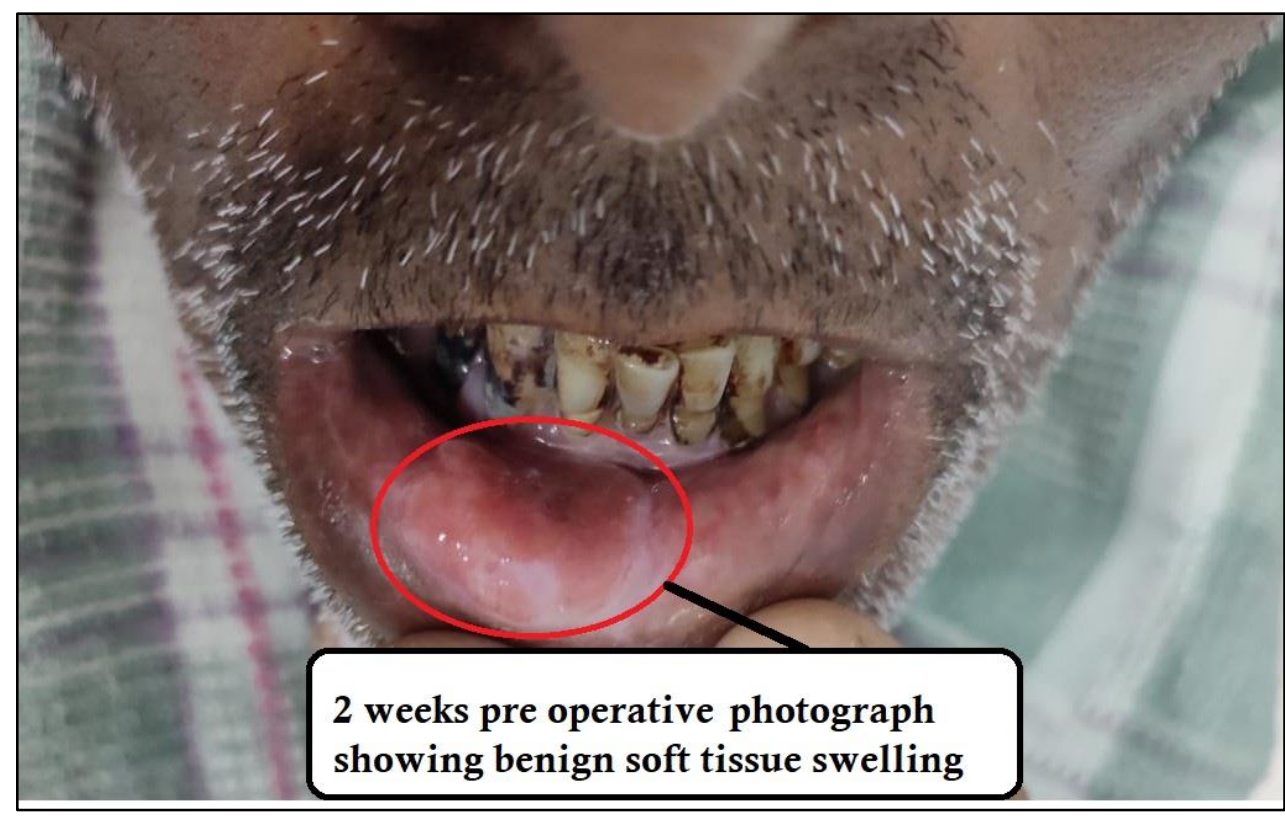

Fig.1 showing 2weeks pre-operative photograph og benign painless, non-tender gradually-growing soft tissue swelling over lower lip . 


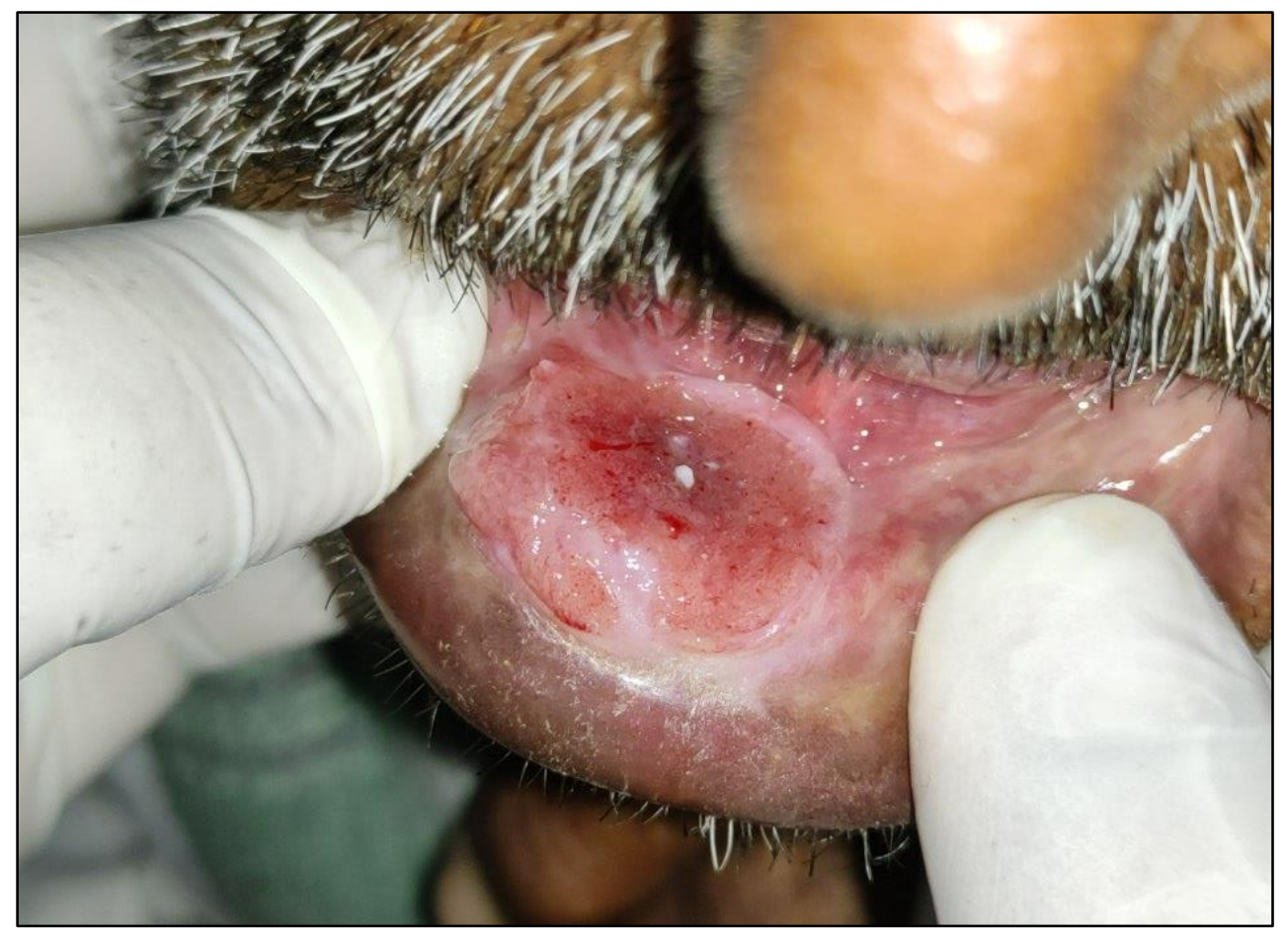

Fig. 2 during examination upon palpation white cheesy material was coming out from the swelling along with erythematous appearance.

Intraoral examination showed that the growth was roughly round, smooth, erythematous, elevated swelling approximately $20 \times 12 \mathrm{~mm}$ in size. Upon palpation white cheesy material expulsion was reported (Fig.2). The patient had grade II oral sub-mucous fibrosis; fibrotic band appeared bilaterally anteriorly from buccinator muscle posterity to retromolar trigone. Two and half finger breadth mouth opening was seen. Clinically, the lesion mimicked benign soft tissue growth hence a provisional diagnosis of traumatic fibroma were made based upon the history and clinical findings.

Differential diagnosis included musicale, haemangioma, lymphangioma, Papiloma, Squamous cell carcinoma. Excisional biopsy was planned and investigations were carried out before surgery. No abnormal radiolucency or radiopacity were detected on orthopantogram in relation to the condition. An excisional biopsy was performed under local anesthesiasia using mepevacaine HCL 2\% and epinephrine as Levonordefrin 1:200,000. The proliferated growth was excised (Fig.5) and sent for histopathological examination. The patient was recalled for follow-up after one week. Healing was found to be unsatisfactory on follow-up. The histopathology report showed nest of malignant squamous epithelial cells arising from epidermis and extending into the dermis (Fig.3). The malignant cells were large pleomorphic with abundant eosinophilic cytoplasm and a large, often vesicular nucleus, variable keratinisation (keratin pearls) \& atypical mitosis was also seen (Fig.4). Stroma showed chronic inflammatory infiltrate suggestive of "Invasive well differentiated squamous cell carcinoma". 


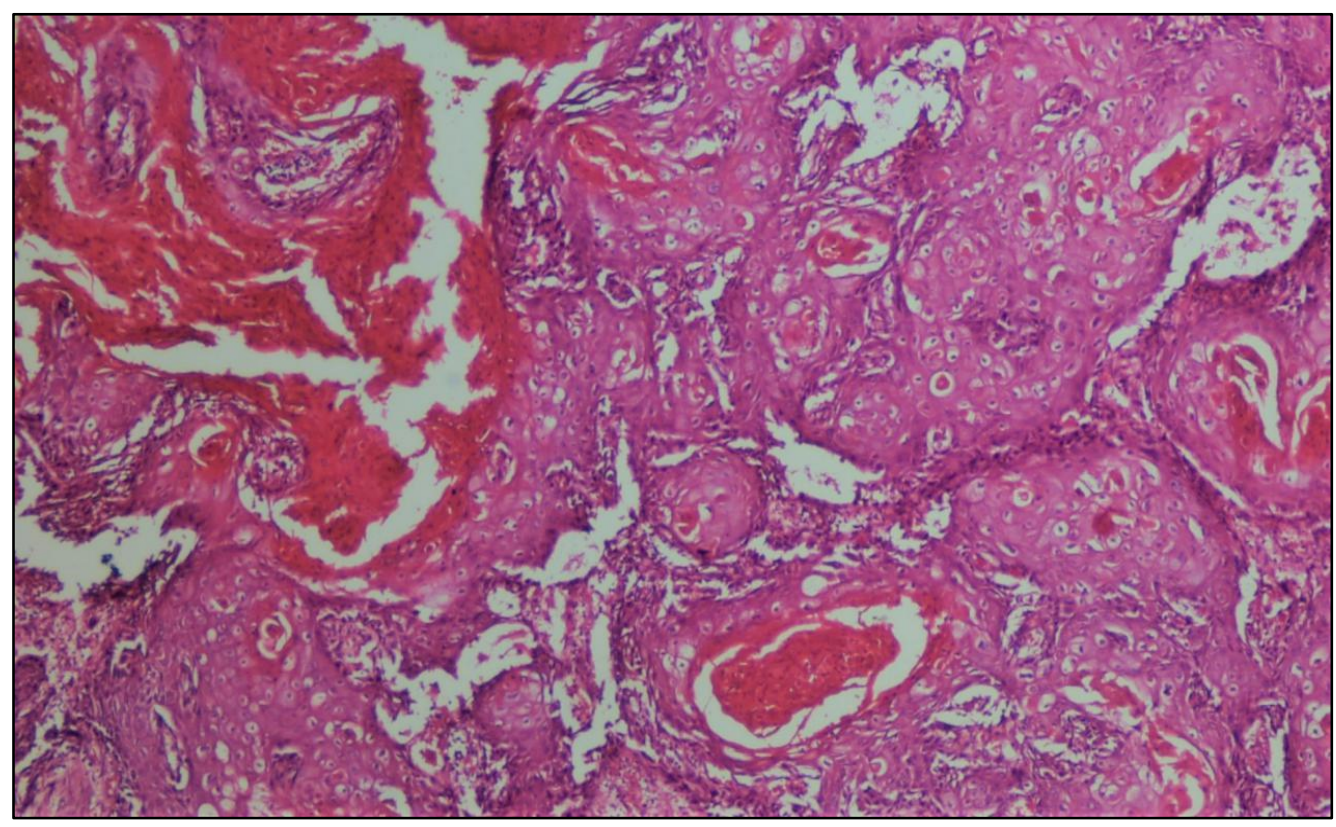

Fig.3 High power photomicrograph showing nest of malignant squamous epithelial cells arising from epidermis and extending into the dermis. indicating invasive squamous cell carcinoma.

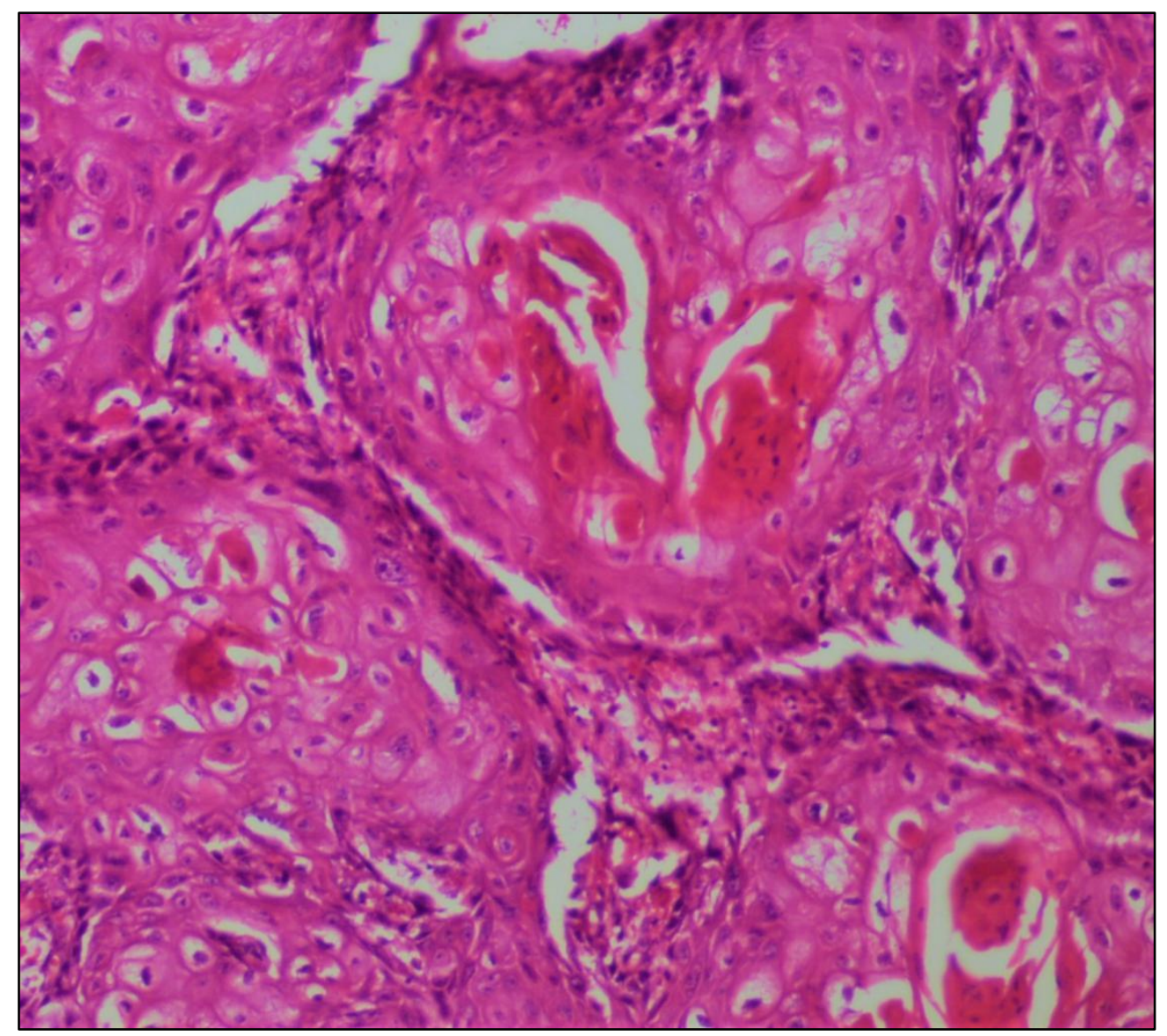

Fig.4 Photomicrograph showing hyperchromatism, altered neuclear cytoplasmic ratio, atypical mitosis, dyskeratotic cells (H\&E stain, x100) 


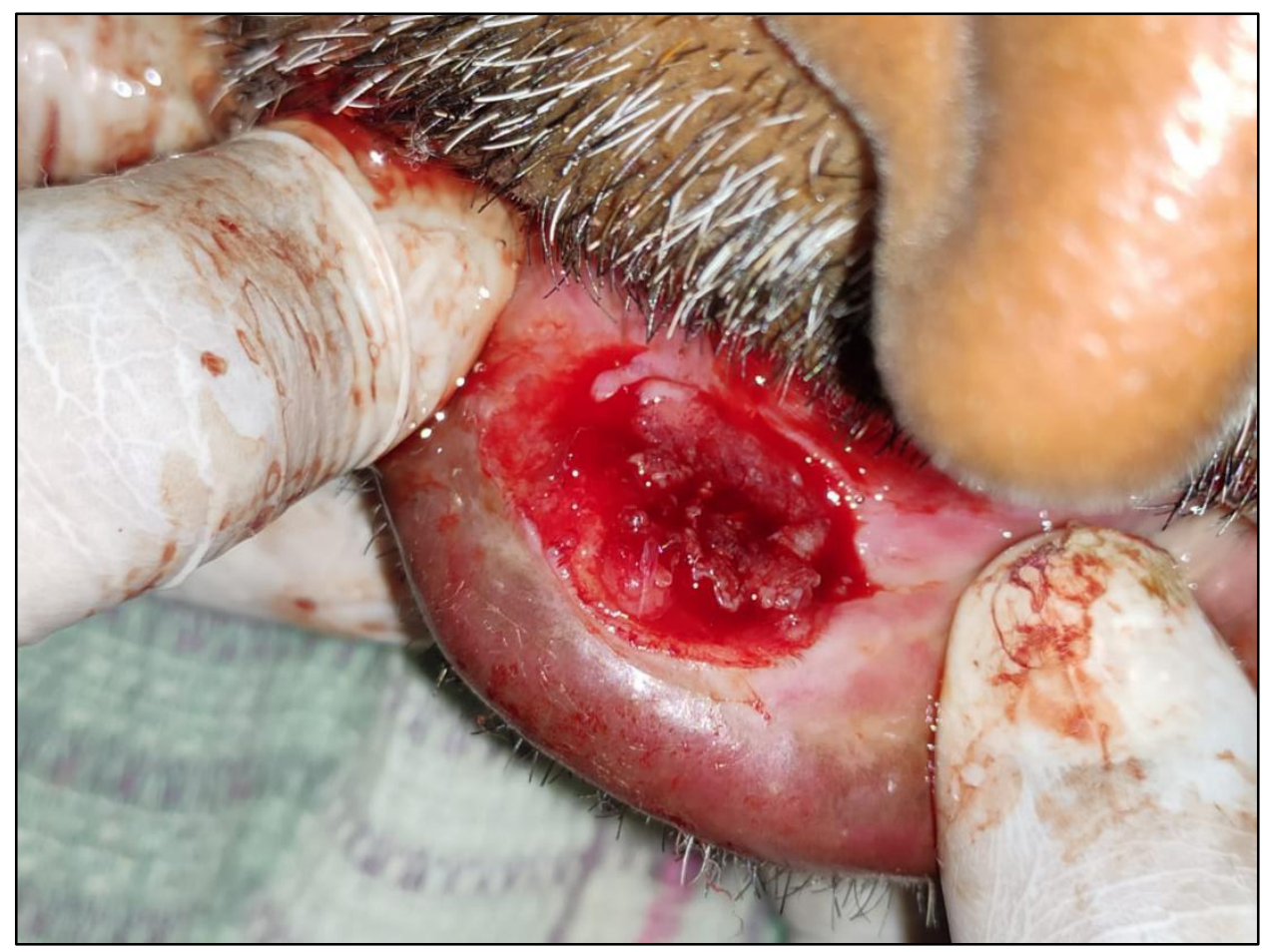

Fig.5 Intra-operative appearance of lower lip after removal of lesion

After confirmation of SCC of the lip by histopathological examination, the patient underwent wedge resection of the entire lesion along with supra-omohyoid neck dissection (SOHND). The reconstruction of lip was done using advancement of the two edges of the defect and re-approximation of the muscle followed by the mucosa and skin under general anaesthesia.

\section{Discussion}

Squamous cell carcinoma of lip occurs at myriad locations along the upper or lower lip or commissure of lip, but the majority ( $80 \%$ to $95 \%$ ) of cases are in the lower lip region (Burusapat\& Pitiseree, 2012). Multiple etiologies are responsible for development of malignancies of lip. These factors can be chronic exposure to solar radiation, tobacco chewing, alcohol consumption, viral etiology (Human Papilloma Virus (HPV) 16 and 24, Herpes Virus (HSV 1 and 2, Epstein-Barr virus), autoimmune diseases and the use of immunological suppressant drugs, whereas possible risk factors includes age (especially 6070 years old) and male sex etc.( Nguyen et al., 2018).

The possible aetiology and risk factors associated with the current case study were tobacco chewing and alcohol consumption, age more than 45 years and male sex. The results of worldwide comparative studies done by Moore et al. (1999) revealed that major unequal regional distribution in the frequency of lip SCC. High incidence rates have been found among the male population of North America, Europe and Oceania, while the frequency of SCC is rare in Asia (Moore et al., 1999). Occurrence of squamous cell carcinoma of lip among Indian population is uncommon and hence the presented case report is a rare phenomenon. Tumour size determines whether tumour will develop into high or low risk 
group by virtue of recurrence and regional metastasis of lip SCC. As per the review of literature, the possibility for regional metastases increases 2.8 folds with the increase of lip SCC size over $2 \mathrm{~cm}$ in diameter (Golubović et al., 2010).

Surgery plays a crucial role in the management of SCC of lip. The goals of surgery are full-thickness resection, lymph node dissection followed by immediate reconstruction. Surgical methods depend mainly on the location and size of tumour. Clinically, visible epithelial cancers of the vermilion border of the lip have a distinctive cauliflower-like exophytic or ulcerated, endophytic nodular surface. They can show myriad grades of underlying musculature infiltration and invasion of the outer skin and/or labial mucosa (Shah $\&$ Singh, 2012). In this given case report, proliferative growth does not show any above mentioned characteristic of neoplastic growth, instead there is mimicking benign soft tissue swelling. Thus, it makes an unusual appearance of Carcinoma of lip.

Lesions that include up to one third of the lower lip are typically treated with V-type or W-type excisions, Lesions affecting about one to two thirds of the lower lip are typically treated by regional flaps of Abbe or Estlander. Larger lesions involving more than two thirds of the lower lip are treated with Bernard flap for middle lesions and nasolabial transpositional flap for lateral lesions (Hasson, 2008).

In this patient, we evaluated that the tumour involves $<2 / 3$ of the lower lip, thus the choice of reconstruction by regional flap, advancement of the two edges of the defect and reapproximation of the muscle followed by the mucosa and skin. Alternatively definitive radiotherapy is equally effective as surgery in local control of early-stage epithelial malignancies of the lips such as basal cell and squamous cell carcinomas. External beam radiation therapy with electrons generally is used for lesions confined to the skin and soft tissues of the lips. Side effects of irradiation include skin dryness, soft tissue atrophy, and indentation of the vermilion border, thus limiting its use in long-term resulting probably in poor prognosis. Therefore, radiotherapy should be reserved for selected situations (Shah \& Singh, 2012).

In vivo study by A. Kutluhan et al.(2003), it is revealed that if elective SOHND is not done and cervical metastasis appears subsequently in lower lip carcinoma, the prognosis worsens substantially and the chance of cure is very small even with salvage surgery and radiation therapy. Thus local resection along with elective SOHND is mandatory for longterm survival rate and to avoid substantial worsening prognosis. In the present case report, sub-mandibular lymph nodes were palpable hence we decided to perform wedge resection along with supra omohyoid neck dissection (SOHND).

\section{Conclusion and Suggestion}

Squamous cell carcinoma is not only the most prevalent malignancy of lip, but it is also one of the most common sites of head and neck squamous cell carcinoma. The classic presentation of squamous cell carcinoma includes chronic non-healing ulcer but it may mimick benign soft tissue growth such as traumatic fibroma, papiloma etc. Therefore, clinicians should be more aware of lesions that do not usually react to routine treatment and thus should be biopsied. 


\section{Ethical Considerations}

For ethical consideration, consent was obtained from the participant in this study.

\section{Conflict of Interest}

The authors of the article declare no conflict of interest.

\section{Funding:}

The authors received no funding for conducting this study.

\section{References}

Burusapat, C., \& Pitiseree, A. (2012). Advanced squamous cell carcinoma involving both upper and lower lips and oral commissure with simultaneous reconstruction by local flap: a case report. Journal of medical case reports, 6(1), 1-7.

Hasson, O. (2008). Squamous cell carcinoma of the lower lip. Journal of Oral and Maxillofacial Surgery, 66(6), 1259-1262.

Shafer WG, Hine MK, Levy MB (2006). A textbook of Oral Pathology, 5th ed. WB Saunders, Philadelphia. pp. 142-163.

Duzgun, S., Ünlu, E., Pekdemir, I., \& Yilanci, S. (2013). Management of squamous cell carcinoma of the lower lip: Analysis of five years' experience (78 patients) and review of the literature. In KBB-Forum.Vol. 12, pp. 26-31.

Zitsch III, R. P., Lee, B. W., \& Smith, R. B. (1999). Cervical lymph node metastases and squamous cell carcinoma of the lip. Head \& Neck: Journal for the Sciences and Specialties of the Head and Neck, 21(5), 447-453.

Salgarelli, A. C., Sartorelli, F., Cangiano, A., Pagani, R., \& Collini, M. (2009). Surgical treatment of lip cancer: our experience with 106 cases. Journal of oral and maxillofacial surgery, 67(4), 840-845.

Nguyen, H. X., Van Nguyen, H., Nguyen, H. X., \& Van Le, Q. (2018). Lower lip squamous cell carcinoma: A Vietnamese case report of surgical treatment with reconstruction by local flap. International journal of surgery case reports, 53, 471474. https://doi.org/10.1016/j.ijscr.2018.11.025

Moore, S. R., Johnson, N. W., Pierce, A. M., \& Wilson, D. F. (1999). The epidemiology of lip cancer: a review of global incidence and aetiology. Oral diseases, 5(3), 185-195.

Golubović, M., Ašanin, B., Jelovac, D., Petrović, M., \& Antunović, M. (2010).

Correlation between disease progression and histopathologic criterions of the lip squamous cell carcinoma. Vojnosanitetski pregled, 67(1), 19-24.

Shah, J. P., Patel, S. G., \& Singh, B. (2012). Head and neck surgery and oncology. Elsevier Health Sciences.

Kutluhan, A., Kiriş, M., Kaya, Z., Kisli, E., Yurttaş, V., Icli, M., \& Kösem, M. (2003). Squamous cell carcinoma of the lower lip and supra-omohyoid neck dissection. Acta Chirurgica Belgica, 103(3), 304-308. 